\title{
Module Count-Based Overflow-Control Scheme for UMTS High-Speed Downlink Packet Access
}

\author{
Phone Lin, Member, IEEE, Yi-Bing Lin, Fellow, IEEE, and Imrich Chlamtac, Fellow, IEEE
}

\begin{abstract}
In the universal mobile telecommunication system, the user equipment (UE) communicates with all cells in the active set through the air interface. Multiple radio links between the UE and the cells may reduce the transmission speed due to interference. In high-speed downlink packet access (HSDPA), only one serving cell is selected in the active set for high-speed downlink transmission. When the radio link quality between the serving cell and the UE degrades below some threshold, the best cell (in terms of the radio characteristics) in the active set is selected as the new serving cell and the UE switches from the old serving cell to the new serving cell. This action is referred to as frame synchronization. The frame-synchronization information may be delivered through more than one wireless transmission, which introduces long delay for the frame-synchronization process. In this paper, we propose an overflow-control scheme with module count for HSDPA, which guarantees that the frame-synchronization information is delivered through one wireless transmission and that when the UE switches wireless link to the new serving cell, no packet frames are lost.
\end{abstract}

Index Terms-High-speed downlink packet access (HSDPA), module count, overflow control, universal mobile telecommunication system (UMTS), UMTS terrestrial radio-access network (UTRAN).

\section{INTRODUCTION}

$\mathbf{U}$ NIVERSAL MOBILE telecommunication services (UMTS) [7] is designed to support high-speed wireless data transmission, which provides streaming, interactive, and background services with better quality of services as compared with the second-generation mobile networks. To further increase the speed of downlink transmission, feasibility studies have been conducted by the Third-Generation Partnership

Manuscript received August 2, 2003; revised November 10, 2003 and December 14, 2003. The work of P. Lin was supported in part by the National Science Council (NSC), R.O.C., under Contract NSC92-2213-E-002-094 FarEastone; Computer \& Communications Researches Laboratories/Industrial Technology Research Institute (CCL/IRTI); and the Institute for Information Industry. The work of Y.-B. Lin was supported in part by the NSC Program for Promoting Academic Excellence of Universities, the Chair Professorship of Providence University; the Institute of Information Science (IIS)/Academia Sinica; FarEastone; CCL/ITRI, and the Lee and MTI Center for Networking Research/National Chiao Tung University (NCTU), R.O.C.

P. Lin is with the Department of Computer Science and Information Engineering, National Taiwan University, Taipei 106, Taiwan, R.O.C. (e-mail plin@csie.ntu.edu.tw).

Y.-B. Lin is with the Department of Computer Science and Information Engineering, National Chiao Tung University, Hsinchu 30050, Taiwan, R.O.C., and with Providence University, Taichung, Taiwan, 43301, R.O.C. (e-mail liny@csie.nctu.edu.tw).

I. Chlamtac is with the Center for Advanced Telecommunications Systems and Services, University of Texas at Dallas, Richardson, TX 75080 USA (e-mail: chlamtac@utdallas.edu).

Digital Object Identifier 10.1109/TVT.2004.823495
Project (3GPP) [1]-[4]. Furthermore, several studies have been contributed to support high-speed downlink packet access (HSDPA) [8]-[10], [12], [14]. In these studies, the major enhancements include adaptive modulation and coding (AMC), fast hybrid automatic repeat request (FHARQ), and fast cell selection (FCS). In this paper, we will focus on the overflow issue that may be caused by FCS.

As shown in Fig. 1, UMTS terrestrial radio access network (UTRAN) consists of node Bs and radio network controllers (RNCs). An RNC is connected to several node Bs through an asynchronous transfer mode (ATM) network [Fig. 1(a)]. To access the UMTS services, a user equipment (UE) communicates with cells (node Bs) in an active set through the air interface Uu based on the wide-band code-division multiple-access (WCDMA) technology [Fig. 1(b)] [5] and a cell may serve several UEs at the same time. For every UTRAN communication session of a UE, an active set of cells is defined [6]. If the quality of the wireless link between the UE and a cell is above some signal strength threshold $\theta_{1}$, this cell is included in the active set. When the quality of the wireless link of a cell in the active set is below another threshold $\theta_{2}$ (where $\theta_{2}<\theta_{1}$ ), the cell is removed from the active set. In a standard UTRAN data session, the UE communicates with all cells in the active set. This approach can not support high-speed downlink transmission because multiple wireless links between several cells and a UE may increase the overall interference within the UTRAN.

3GPP TS 25.308 [4] and TR 25.950 [3] proposed an approach to support HSDPA [2]-[4], where a UE only communicates with one cell (called the serving cell) in the active set and a serving cell may serve different UEs. This serving cell is selected by the fast cell-selection procedure [2] based on the common pilot-channel received signal-code power measurements of the cells in the active set. Two physical channels, high-speed physical-downlink shared channel (HS-PDSCH) and dedicated physical-control channel (DPCCH) are used for downlink packet-frame transmission and uplink/downlink signaling, respectively. While multiple cells may be members in the active set, only one transmits packet frames to the UE at any time in the HSDPA mode. Therefore, the interference within a cell is potentially decreased and the system capacity is increased. In HSDPA, the RNC sends packet frames to all cells in the active set. Each of the nonserving cells queues the packet frames in a buffer. On the other hand, the serving cell forwards the packet frames to the UE. The stop-and-wait hybrid automatic repeat request (SAW-hybrid ARQ) [17] algorithm is exercised between the UE and the serving cell for flow control of wireless transmission. If the link quality for current 


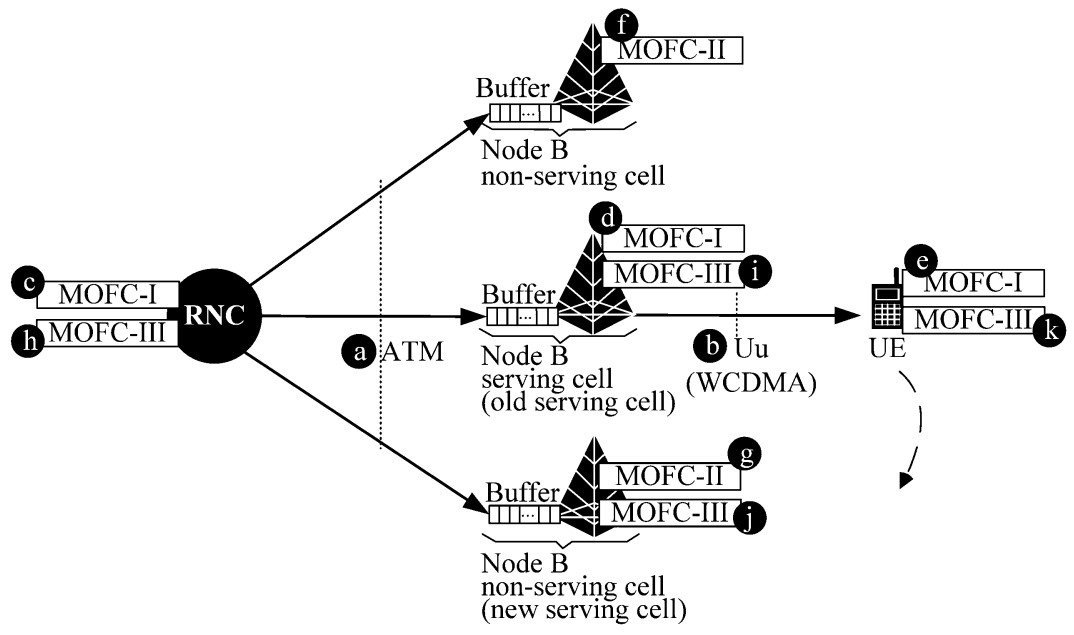

Fig. 1. UTRAN architecture.

high-speed downlink transmission degrades below a threshold $\theta_{2}$, the cell with the best link quality in the active set is selected by the network as the new serving cell. Then, the next packet frames are transmitted from the new serving cell to the UE. In HSDPA, two issues must be addressed.

Buffer Overflow Issue: The buffer in a nonserving cell may be full and a mechanism is required to avoid buffer overflow at that cell.

Frame Synchronization Issue: When the UE switches to a new serving cell for downlink packet access, the new serving cell should be informed of the status of the buffer (i.e., the number of packet frames received by the UE) in the old serving cell and appropriately drop the redundant packet frames in the buffer. This action is referred to as frame synchronization [3].

Our previous study proposed schemes to resolve the above two issues. Details can found in [15] and [16]. In the previously proposed schemes, the information needed for frame synchronization is carried by the uplink DPCCH. When the size of frame-synchronization information exceeds the capacity of one uplink DPCCH message, this information must be carried through multiple uplink DPCCH messages. In this paper, we propose module count-based overflow control (MOFC) to guarantee that the frame-synchronization information is delivered through one uplink DPCCH message. This paper is organized as follows. First, we describe the MOFC scheme in Section II. Then, we prove that the proposed scheme functions correctly in Section III. Finally, we compare the performance of MOFC with the previously proposed schemes.

\section{Module-Count-Based Overflow-Control Scheme}

The MOFC scheme consists of three procedures: MOFC-I, MOFC-II, and MOFC-III. The relationship among these procedures is shown in Fig. 1. By exercising MOFC-I, the packet frames are delivered from the RNC to the UE through the serving cell Cell $s$ [see $(c),(d)$, and $(e)$ ]. MOFC-II is executed by a nonserving cell to avoid buffer overflow [see $(f)$ and $(g)$ ]. MOFC-III is exercised for frame synchronization when the UE switches the wireless link from the old serving cell to the new serving cell [see $(h),(i),(j)$, and $(k)$ ]. The notation used in this paper is listed in the Appendix. Details of MOFC-I, MOFC-II, and MOFC-III are described as follows.

\section{A. MOFC-I and MOFC-II}

In HSDPA, the RNC sends packet frames to all node Bs in the active set. The serving cell forwards the packet frames to the UE. On the other hand, the nonserving cells execute MOFC-II to buffer the received packet frames without actually sending them to the UE. Let $N_{i}$ be the buffer size of Cell ${ }_{i}$. Fig. 2 illustrates the timing diagram for MOFC-I, which consists of two parts: Part 1 is exercised between the RNC and Cell $s$ and Part 2 is exercised between $\mathrm{Cell}_{s}$ and the UE.

Part 1: A window-based flow-control algorithm with window size $w$ is exercised between the $\mathrm{RNC}$ and $\mathrm{Cell}_{s}$. Since the ATM link [see Fig. 1(a)] is considered to be reliable, we assume that no packet frame is lost or corrupted during transmission. Therefore, we do not consider packet frame retransmission at the ATM link. If the rare events of packet-frame loss do occur, these lost packet frames can be recovered by higher level protocols.

The RNC maintains a counter $C S_{\mathrm{RNC}}$ to record the number of packet frames that have been transmitted to the cells in the active set. Every time the RNC sends a packet frame [see Fig. 2(a)], it increments $C S_{\mathrm{RNC}}$ by one.

After transmitting $w$ packet frames, the RNC suspends the transmission until it receives an $\mathrm{ack}_{s}$ message sent from $\mathrm{Cell}_{s}$ [see Fig. 2(b)]. The $\mathrm{ack}_{s}$ message allows the RNC to transmit the packet frames of the next window. A counter $C R_{s}$ is maintained by $\mathrm{Cell}_{s}$ to count the number of packet frames received from the RNC. Another counter $C S_{s}$ is maintained by Cell ${ }_{s}$ to record the number of packet frames sent to the UE. When a packet frame arrives at Cell $s, \mathrm{Cell}_{s}$ stores the packet frame at the tail of the buffer and increments $C R_{s}$ by one.

After all packet frames in a window have been received, Cell ${ }_{s}$ computes the number $K_{s}$ of packet frames currently stored in the buffer, where $K_{s}=C R_{s}-C S_{s}$. Cell ${ }_{s}$ checks if there is free space to accommodate the packet frames 


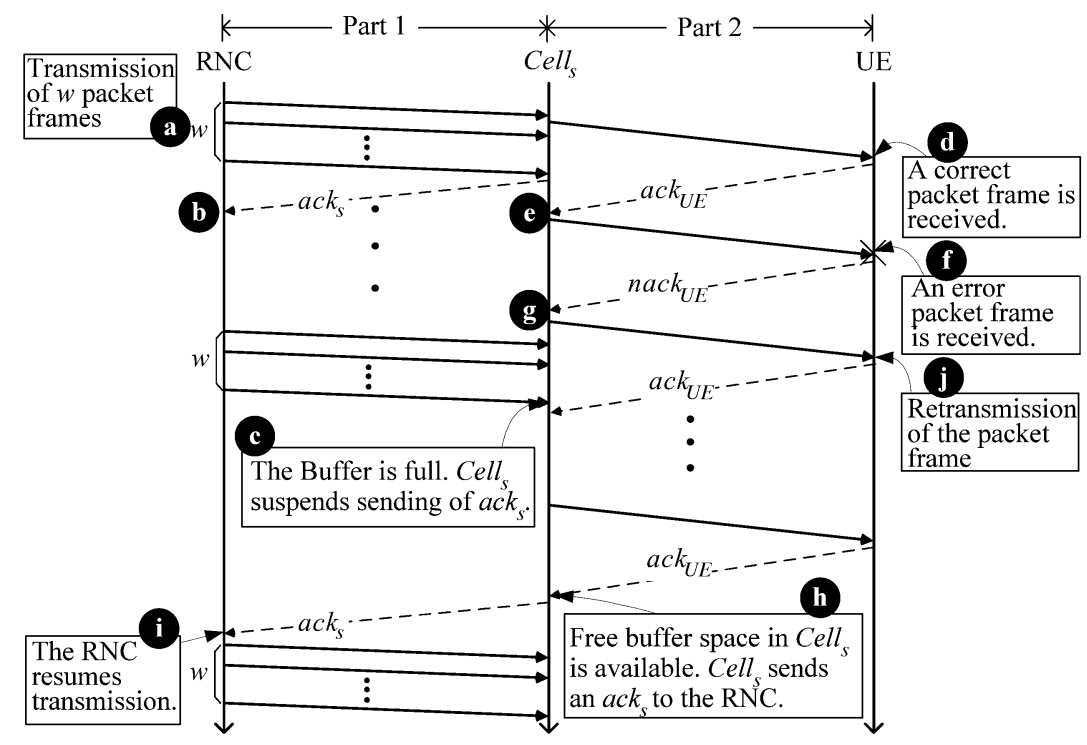

Fig. 2. Timing diagram for MOFC-I.

of the next window and determines if it should send the $\mathrm{ack}_{s}$ message to the RNC. The window-based flow-control mechanism is described by considering the $K_{s}$ value in the following two cases.

Case MOFC-I.1. $K_{s} \leq N_{s}-w$ : Cell $_{s}$ sends ack to the RNC, which allows the RNC to deliver the packet frames of the next window [see Fig. 2(b)].

Case MOFC-I.2. $K_{s}>N_{s}-w$ : No action is taken. In this case, $\mathrm{Cell}_{s}$ does not send $\mathrm{ack}_{s}$ to the RNC and the subsequent packet-frame transmission is suspended [see Fig. 2(c)].

The RNC is allowed to send next packet frames only in Case MOFC-I.1 (when $K_{s} \leq N_{s}-w$ ). This restriction ensures that Cell ${ }_{s}$ has enough buffer space to accommodate the packet frames of the next window.

Part 2: The SAW-hybrid ARQ flow-control algorithm [17] is exercised between the UE and $\mathrm{Cell}_{s}$ [see Fig. 1(b)]. Since errors may occur during unreliable wireless transmission, the packet frames received by the UE may be incorrect. Two types of acknowledgment messages, ack $\mathrm{UE}_{\mathrm{E}}$ and nack $\mathrm{UE}_{\mathrm{E}}$, will be sent from the UE to $\mathrm{Cell}_{s}$, which indicate if the UE receives correct or error-packet frames from $\mathrm{Cell}_{s}$, respectively. Each time, $\mathrm{Cell}_{s}$ sends a packet frame to the UE and waits for an acknowledgment from the UE. The UE maintains a counter $C R_{\mathrm{UE}}$ to record the number of packet frames that have been correctly received from $\mathrm{Cell}_{s}$. If the UE receives a correct packet frame from $\mathrm{Cell}_{s}$ [see Fig. 2(d)], it increments $C R_{\mathrm{UE}}$ by one. Then, the UE sends $\operatorname{ack}_{\mathrm{UE}}$ to $\mathrm{Cell}_{s}$ [see Fig. 2(e)]. If an error-packet frame is received [see Fig. 2(f)], the UE replies Cell $_{s}$ a nack ${ }_{U E}$ message [see Fig. 2( $g)$ ].

If $\mathrm{Cell}_{s}$ receives ack $\mathrm{UE}_{\mathrm{E}}$ from the $\mathrm{UE}$, it increments $C S_{s}$ by one and drops the transmitted packet frame. If $\mathrm{Cell}_{s}$ receives nack ${ }_{U E}$, it retransmits the packet frame [see Fig. 2(j)]. After successfully sending a packet frame to the $\mathrm{UE}, \mathrm{Cell}_{s}$ determines if it should send $\mathrm{ack}_{s}$ to the RNC by checking the number of packet frames currently stored in the buffer (i.e., $K_{s}$ ). Two cases are considered, as follows.

Case MOFC-I.3. If $\mathrm{Cell}_{s}$ has not suspended the transmission of $\mathrm{ack}_{s}$, no action is taken.

Case MOFC-I.4. If $\mathrm{Cell}_{s}$ has suspended the transmission of $\mathrm{ack}_{s}$, it calculates the number $K_{s}$ of packet frames currently stored in the buffer; specifically, $K_{s}=C R_{s}-C S_{s}$. If $K_{s}>N_{s}-w$, Cell $s$ takes no action (i.e., the buffer space is not large enough and the transmission from the RNC to $\mathrm{Cell}_{s}$ is still suspended). On the other hand, if $K_{s} \leq N_{s}-w$, Cell $s$ sends ack to the RNC to trigger packet-frame delivery of the next window [see Figs. 2( $h)$ and $(i)$ ].

Note that the variable $K_{s}$ can be accessed and updated by parts 1 and 2 of MOFC-I. If these two parts are executed in parallel, then the accesses and modifications to $K_{s}$ must be atomic operations. Most likely, parts 1 and 2 of MOFC-I are alternatively executed in sequence.

Every nonserving cell $\mathrm{Cell}_{i}$ exercises MOFC-II to avoid buffer overflow. In $\mathrm{Cell}_{i}$, two counters $C R_{i}$ and $C S_{i}$ are maintained to record the numbers of packet frames that have been received from the RNC and deleted by $\mathrm{Cell}_{i}$, respectively. When Cell $_{i}$ receives a packet frame from the RNC, it recalculates $K_{i}$ as $C R_{i}-C S_{i}$. Two cases are considered, as follows.

Case MOFC-II.1. If $K_{i}<N_{i}$ (i.e., the buffer has enough space to accommodate the incoming packet frame), Cell adds the received packet frame at the tail of the buffer, and increments $C R_{i}$ by one.

Case MOFC-II.2. If $K_{i}=N_{i}$ (i.e., the buffer is full), Cell deletes a packet frame at the head of the buffer and increments $C S_{i}$ by one. Cell $i$ adds the newly received packet frame at the tail of the buffer and increments $C R_{i}$ by one. This case guarantees that the buffer of $\mathrm{Cell}_{i}$ always has space to accommodate the incoming packet frame. In Section II-B, we will show that every old packet frame deleted in this step is already received by the UE. 


\section{B. Determination of Buffer Sizes}

In MOFC, if the radio link quality between the serving cell and UE degrades below the threshold $\theta_{2}$, the best cell in the active set is selected as the new serving cell. To switch the wireless transmission link from the old serving cell Cell $s$ to the new serving cell Cell $i$, frame synchronization is required. The frame-synchronization information, i.e., the $C R_{\mathrm{UE}}$ value, is sent from the UE to $\mathrm{Cell}_{i}$. Then, $\mathrm{Cell}_{i}$ computes $N_{\text {sync }}$, the number of packet frames that have been received by the UE but are still in Cell $i$ 's buffer. That is

$$
N_{\mathrm{sync}}=C R_{\mathrm{UE}}-C S_{i} .
$$

When Cell $i$ becomes the serving cell, it deletes the first $N_{\text {sync }}$ packet frames in the buffer and then transmits the next packet frames in the buffer to the UE. Note that $C R_{\mathrm{UE}}$ should always be no less than $C S_{i}$. Otherwise (i.e., $C R_{\mathrm{UE}}<C S_{i}$ ), it means that $\mathrm{Cell}_{i}$ has dropped packet frames that have not been received by the UE. These packet frames are lost when the UE switches the wireless link to $\mathrm{Cell}_{i}$. Consider two constants: $B_{1}$ and $B_{2}$. In [16], we proved the following theorem.

Theorem 1: Let the buffer sizes of the serving cell $\mathrm{Cell}_{s}$ and every nonserving cell Cell ${ }_{i}$ be $N_{s}=B_{1}$ and $N_{i}=B_{2}$, respectively. If $B_{1} \leq B_{2}-w$, then when MOFC-I and MOFC-II are exercised, it is guaranteed that $C R_{\mathrm{UE}}$ is always no less than $C S_{i}$.

Following Theorem 1 , we set $B_{1}=B_{2}-w$ in MOFC so that the nonserving cells can safely delete the old packet frames in the buffer. Another issue to be addressed by MOFC is the avoidance of multiple DPCCH message delivery. At frame synchronization, the $C R_{\mathrm{UE}}$ value may be larger than the number that can be carried in one uplink DPCCH message. If so, the $C R_{\mathrm{UE}}$ value must be delivered through multiple DPCCH messages [15], [16], which results in a long frame-synchronization delay. To resolve this issue, MOFC uses the module operation to tailor the $C R_{\mathrm{UE}}$ value to a smaller value $C R_{\mathrm{UE}}^{*}$, which can be accommodated in one uplink DPCCH message. After receiving the $C R_{\mathrm{UE}}^{*}$ value, the nonserving cell $\mathrm{Cell}_{i}$ determines $N_{\text {sync }}$ based on Theorem 2, described as follows.

Theorem 2: Let $x$ be a nonnegative integer. Let $C R_{\mathrm{UE}}^{*}=$ $C R_{\mathrm{UE}} \bmod x$ and $C S_{i}^{*}=C S_{i} \bmod x$. If

$$
0 \leq C R_{\mathrm{UE}}-C S_{i}<x
$$

then

$$
N_{\mathrm{sync}}=C R_{\mathrm{UE}}-C S_{i}=\left\{\begin{array}{c}
C R_{\mathrm{UE}}^{*}-C S_{i}^{*} \\
\text { if } C R_{\mathrm{UE}}^{*}-C S_{i}^{*} \geq 0 . \\
x+C R_{\mathrm{UE}}^{*}-C S_{i}^{*} \\
\text { if } C R_{\mathrm{UE}}^{*}-C S_{i}^{*}<0 .
\end{array}\right.
$$

Proof: Let $a=\left\lfloor C R_{\mathrm{UE}} / x\right\rfloor$ and $b=\left\lfloor C S_{i} / x\right\rfloor$. Then, it is apparent that

$$
C R_{\mathrm{UE}}=a x+C R_{\mathrm{UE}}^{*}
$$

and

$$
C S_{i}=b x+C S_{i}^{*}
$$

where both $a$ and $b$ are nonnegative integers. By subtracting (2) from (3), we have

$$
C R_{\mathrm{UE}}-C S_{i}=(a-b) x+\left(C R_{\mathrm{UE}}^{*}-C S_{i}^{*}\right)
$$

From (1) and (4), we have

$$
0 \leq(a-b) x+\left(C R_{\mathrm{UE}}^{*}-C S_{i}^{*}\right)<x .
$$

Since $0 \leq C R_{\mathrm{UE}}^{*}<x$ and $0 \leq C S_{i}^{*}<x, C R_{\mathrm{UE}}^{*}-C S_{i}^{*}$ is bounded in the range

$$
-x<C R_{\mathrm{UE}}^{*}-C S_{i}^{*}<x .
$$

Based on Inequality (6), we consider the following two cases.

Case I: If $C R_{\mathrm{UE}}^{*}-C S_{i}^{*} \geq 0$, then from (6) we have $0 \leq$ $C R_{\mathrm{UE}}^{*}-C S_{i}^{*}<x$. Consider the relationship between $a$ and $b$. If $a>b$, then $(a-b) x+\left(C R_{\mathrm{UE}}^{*}-C S_{i}^{*}\right) \geq x$, which contradicts (5). If $a<b$, then $(a-b) x+\left(C R_{\mathrm{UE}}^{*}-C S_{i}^{*}\right)<$ 0 , which also contradicts (5). Therefore, $a$ must be equal to $b$ in this case and (4) can be rewritten as $C R_{\mathrm{UE}}-C S_{i}=$ $C R_{\mathrm{UE}}^{*}-C S_{i}^{*}$.

Case II: If $C R_{\mathrm{UE}}^{*}-C S_{i}^{*}<0$, then from (6), $-x<$ $C R_{\mathrm{UE}}^{*}-C S_{i}^{*}<0$. If $a \leq b$, then $(a-b) x+\left(C R_{\mathrm{UE}}^{*}-\right.$ $\left.C S_{i}^{*}\right)<0$, which contradicts (5). On the other hand, if $a-b \geq 2$, then $(a-b) x+\left(C R_{\mathrm{UE}}^{*}-C S_{i}^{*}\right)>x$, which also contradicts (5). Therefore, $a-b=1$. In this case, (4) is rewritten as $C R_{\mathrm{UE}}-C S_{i}=x+C R_{\mathrm{UE}}^{*}-C S_{i}^{*}$.

The above two cases complete the proof.

In UTRAN, the transmission rate of the wire-line link between the RNC and a node B is much higher than that of the wireless link between the UE and a node B. Since node Bs and the RNCs are connected by ATM links [see Fig. 1(a)], the overall transmission rate is about $50 \mathrm{Mb} / \mathrm{s}$ [11], [13]. On the other hand, a node B and all UEs in its coverage area are connected by the WCDMA radio links [see Fig. 1(b)] and the overall downlink wireless-transmission rate is $3.84 \mathrm{Mb} / \mathrm{s}$ [11]. Let the transmission delays from the RNC to Cell $s$ and $\mathrm{Cell}_{i}$ be $t_{A, 1}$ and $t_{A, 2}$, respectively. Without loss of generality, $t_{A, 1} \approx t_{A, 2}$. Let the transmission delay from $\mathrm{Cell}_{s}$ to the UE be $t_{W}$. Then, from the above description, $t_{W} \gg t_{A, 2}$ and $t_{W}+t_{A, 1} \gg t_{A, 2}$, which implies that if a packet frame is received by the UE, the packet frame is also received by Cell ${ }_{i}$. Thus, for any nonserving cell $\mathrm{Cell}_{i}$, we have $C R_{\mathrm{UE}} \leq C R_{i}$. When a nonserving cell Cell ${ }_{i}$ exercises MOFC-II, if $C S_{i}>0$ (i.e., some packet frames have been dropped), it means that the buffer is full at Cell $_{i}$ and $C R_{i}=C S_{i}+B_{2}$. Therefore, if $C S_{i}>0$, then

$$
C R_{\mathrm{UE}} \leq C S_{i}+B_{2} \quad \text { or } \quad C R_{\mathrm{UE}}-C S_{i}<B_{2}+1
$$

Since we set $B_{1}=B_{2}-w$ in MOFC, from Theorem 1 and (7) we have

$$
0 \leq C R_{\mathrm{UE}}-C S_{i}<B_{2}+1
$$




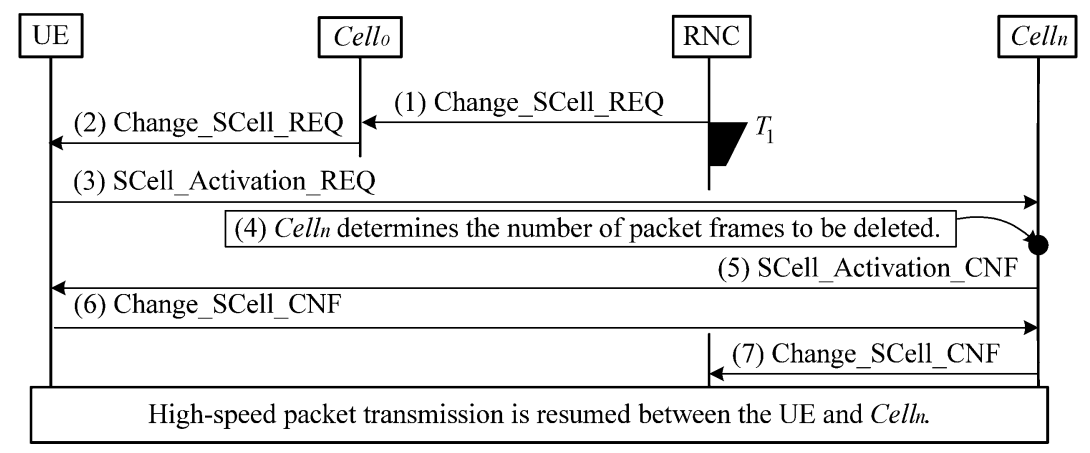

Fig. 3. Message flow for MOFC-III.

If the value of $x$ is $B_{2}+1$, then from Theorem 2 and (8) we have

$$
N_{\mathrm{sync}}=C R_{\mathrm{UE}}-C S_{i}=\left\{\begin{array}{c}
C R_{\mathrm{UE}}^{*}-C S_{i}^{*} \\
\text { if } C R_{\mathrm{UE}}^{*}-C S_{i}^{*} \geq 0 \\
B_{2}+1+C R_{\mathrm{UE}}^{*}-C S_{i}^{*} \\
\text { if } C R_{\mathrm{UE}}^{*}-C S_{i}^{*}<0
\end{array}\right.
$$

where $C R_{\mathrm{UE}}^{*}=C R_{\mathrm{UE}} \bmod \left(B_{2}+1\right)$ and $C S_{i}^{*}=C S_{i} \bmod$ $\left(B_{2}+1\right)$. Then, a nonserving cell can use the $C R_{\mathrm{UE}}^{*}$ value to correctly determine the $N_{\text {sync }}$ value. Thus, we have the following lemma.

Lemma 1: Let $N^{*}$ denote the maximum value of the number that can be accommodated in one uplink DPCCH message. To transmit $C R_{\mathrm{UE}}^{*}$ in one uplink DPCCH message, $C R_{\mathrm{UE}}^{*}=C R_{\mathrm{UE}} \bmod \left(B_{2}+1\right)$ must be no larger than $N^{*}$. That is, the inequality $B_{2}+1 \leq N^{*}$ must hold.

From the above discussion, we can determine $B_{1}$ and $B_{2}$ as follows. We first set $B_{2}=N^{*}-1$. Then, we set $B_{1}=B_{2}-w$.

\section{MOFC-III}

To switch the wireless link from the old serving cell $\mathrm{Cell}_{O}$ to the new serving cell Cell ${ }_{n}$, MOFC-III is executed among the $\mathrm{UE}, \mathrm{Cell}_{o}, \mathrm{Cell}_{n}$ and the RNC for frame synchronization. We utilize Theorem 2 to guarantee that the frame-synchronization information can be delivered in one uplink DPCCH message. Suppose that $b$ bits are used to hold frame-synchronization information in a uplink DPCCH message. In MOFC-III, the value $2^{b}-1$ is used to represent the symbol NULL (which indicates that the UE did not receive any packet frame from the old serving cell $\mathrm{Cell}_{o}$ ). The maximum number that can be accommodated in one DPCCH message is $N^{*}=2^{b}-2$. Fig. 3 illustrates the message flow for MOFC-III. In this message flow, Steps 1, 2, 6, and 7 are defined in Specification 3G TS25.308 [4]. In this document, frame synchronization is left as an open issue. In this paper, we address this issue by adding Steps 3-5. The details are given as follows.

Step 1. Based on the radio quality measurement report sent from the UE, the RNC determines the need of radio link switching for the UE. The RNC first selects a new serving cell Cell ${ }_{n}$ and instructs Cell $_{n}$ to reserve an HS-PDSCH channel for the UE for downlink packet-frame transmission. Then, the RNC sends Cell ${ }_{o}$ a Change_SCell_REQ message (where the ID of the new serving cell Cell ${ }_{n}$ is specified in this message) and starts the $T_{1}$ timer. The RNC expects to receive the Change_SCell_CNF message (see Step 7) before the $T_{1}$ timer expires. If the $T_{1}$ timer expires, the RNC may choose to resend the Change_SCell_REQ message or to exit MOFC-III.

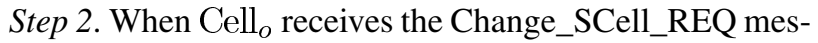
sage, it stops high-speed downlink packet-frame transmission to the UE. Cell $l_{o}$ forwards the Change_SCell_REQ message to the UE, where the ID of $\mathrm{Cell}_{n}$ is contained in the message. Then, Cell $_{o}$ adjusts its buffer size as $N_{o}=B_{2}$ and then executes MOFC-II to process the packet frames received from the RNC. At this point, $\mathrm{Cell}_{O}$ becomes a nonserving cell.

Step 3. Upon receipt of the Change_SCell_REQ message, the UE is aware of the change of the serving cell to Cell ${ }_{n}$. The UE checks the value of $C R_{\mathrm{UE}}$. If no packet frame is received by the $\mathrm{UE}$ (i.e., $C R_{\mathrm{UE}}=0$ ), then the $\mathrm{UE}$ sets $C R_{\mathrm{UE}}^{*} \leftarrow$ NULL. Otherwise (i.e., $C R_{\mathrm{UE}}>0$ ), $C R_{\mathrm{UE}}^{*} \leftarrow C R_{\mathrm{UE}} \bmod \left(B_{2}+1\right)$. The UE sends an SCell_Activation_REQ message to Cell $_{n}$ through the uplink DPCCH (where $C R_{\mathrm{UE}}^{*}$ is specified in this message). Since $B_{2}=N^{*}-1$, the $C R_{\mathrm{UE}}^{*}$ value can be fitted in one uplink DPCCH message.

Steps 4 and 5. Upon receipt of the SCell_Activation_REQ message, $\mathrm{Cell}_{n}$ determines the number of packet frames to be deleted from the buffer for frame synchronization. Based on the value of $C R_{\mathrm{UE}}^{*}$, two cases are considered, as follows.

Case 4.1: $C R_{\mathrm{UE}}^{*}=$ NULL, i.e., the UE did not receive any packet frame before switching the wireless link and no packet frame should be deleted.

Case 4.2: $C R_{\mathrm{UE}}^{*} \neq$ NULL, i.e., the $U E$ has received some packet frames before switching the wireless link. Cell ${ }_{n}$ first tailors $C S_{n}$ to a smaller value $C S_{n}^{*}$; specifically, $C S_{n}^{*}=C S_{n} \bmod \left(B_{2}+1\right)$. If $C R_{\mathrm{UE}}^{*}-C S_{n}^{*} \geq 0$, then Cell $_{n}$ deletes $C R_{\mathrm{UE}}^{*}-C S_{n}^{*}$ packet frames at the head of the buffer. Otherwise (i.e., $C R_{\mathrm{UE}}^{*}-C S_{n}^{*}<0$ ), Cell $_{n}$ deletes $B_{2}+1+C R_{\mathrm{UE}}^{*}-C S_{n}^{*}$ packet frames at the head of the buffer.

Cell $l_{n}$ adjusts its buffer size as $N_{n}=B_{1}$ and then sends the UE an SCell_Activation_CNF message.

Steps 6 and 7. UE is informed that $\mathrm{Cell}_{n}$ has performed frame synchronization when it receives the SCell_Activation_CNF message from $\mathrm{Cell}_{n}$. The UE then sends a Change_SCell_CNF message to the RNC through Cell $_{n}$. 
At this point, $\mathrm{Cell}_{n}$ starts high-speed downlink packetframe transmission to the UE by executing MOFC-I.

\section{CORRECTNESS PROOF FOR FRAME SYNCHRONIZATION}

This section proves the correctness of MOFC-III. In Lemma 2 , we first prove that before frame synchronization, the new serving cell Cell ${ }_{n}$, did not delete any packet frames that have not been received by the UE. Then, from Theorem 2 and Lemmas 1 and 2, Theorem 3 concludes that MOFC-III correctly performs frame synchronization with one uplink DPCCH message delivery.

Lemma 2: Before Step 4 in MOFC-III is executed, the new serving cell Cell ${ }_{n}$ did not delete any packet frames that have not been received by the UE.

Proof: Before Step 4 in MOFC-III is executed, Cell ${ }_{n}$ is a nonserving cell (that executes MOFC-II to avoid buffer overflow). The buffer size of Cell $l_{n}$ is set as $N_{n}=B_{2}$. Since $B_{2}=B_{1}+w$, Theorem 1 guarantees that $C R_{\mathrm{UE}} \geq C S_{n}$ (that is, the number of packet frames received by the UE is larger than those dropped by $\mathrm{Cell}_{n}$ ). Therefore, for the packet frames that have not been received by the UE, none were deleted by the new serving cell Cell $n$.

Theorem 3: MOFC-III correctly performs frame synchronization with one uplink DPCCH message delivery.

Proof: From Lemma 2, Cell $n$ does not drop any packet frames that have not been received by the UE. Lemma 1 guarantees that the $C R_{\mathrm{UE}}^{*}$ value is carried in one uplink DPCCH message as Step 3 in MOFC-III is executed. From Theorem 2, if the UE has received packet frames at Cell (i.e., $\left.C R_{\mathrm{UE}}^{*} \neq \mathrm{NULL}\right)$, Cell $_{n}$ correctly deletes the packet frames that have been received by the UE but are still queued in the buffer of Cell $n$ (see Case 4.2 in MOFC-III). If no packet frames have been received by the $\mathrm{UE}$ at $\mathrm{Cell}_{o}$ (i.e., $C R_{\mathrm{UE}}^{*}=\mathrm{NULL}$ ), $\mathrm{Cell}_{n}$ does not delete any packet frame in its buffer (see Case 4.1 in MOFC-III). Therefore, after MOFC-III is executed, $\mathrm{Cell}_{n}$ correctly transmits the subsequent packet frames to the UE.

\section{NUMERICAL EXAMPLES}

In this section, we elaborate on the improvement of MOFC over several schemes proposed in our previous studies [15], [16], including basic overflow control (BOFC), network-based overflow control (NOFC), and combined BOFC and NOFC (COFC). Details of these schemes and the modeling technique used in this section can be found in [15] and [16]. BOFC, NOFC, and COFC are briefly described as follows.

$B O F C$. In this scheme, if the size of the frame-synchronization information exceeds the capacity of one uplink DPCCH message, the information is carried by multiple uplink DPCCH messages.

NOFC. This scheme guarantees one uplink DPCCH message delivery by exchanging the frame-synchronization information through the network between the old and new serving cells.

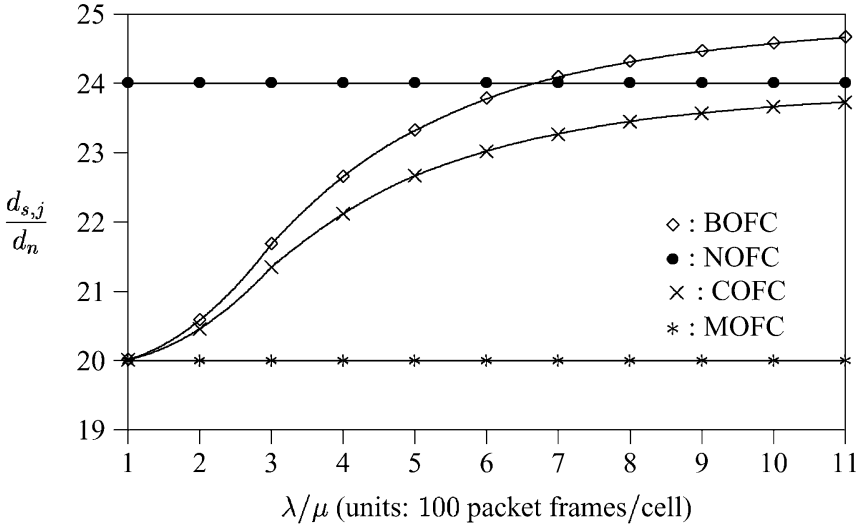

(a)

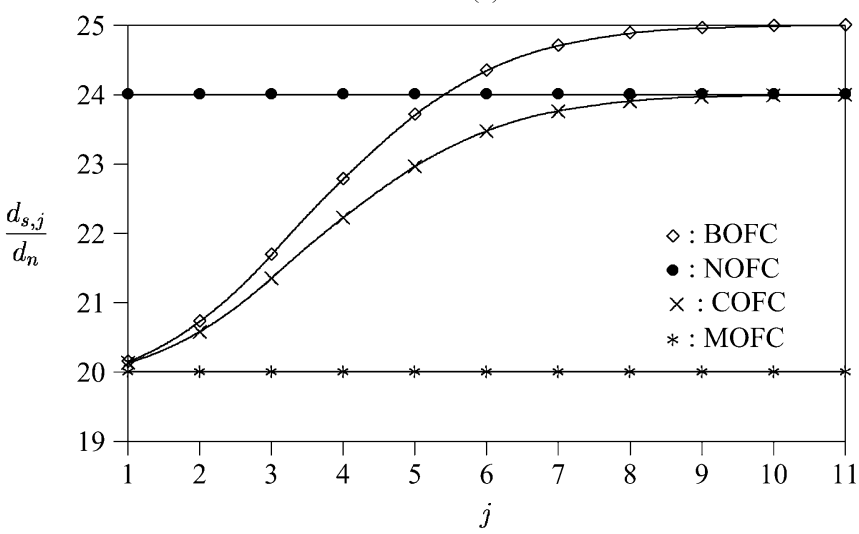

(b)

Fig. 4. Performance comparison among MOFC, BOFC, NOFC, and COFC $\left(d_{r}=5 d_{n} ; N^{*}=1023\right)$. (a) Effects of $\lambda / \mu(j=3)$. (b) Effects of $j(\lambda / \mu=$ $300)$.

COFC. This scheme takes advantage of both BOFC and NOFC. The old serving cell decides whether to transmit frame-synchronization information through the network (using NOFC) or to uplink DPCCH messages (using BOFC).

Consider the net cost $d_{s, j}$ for frame synchronization when the UE switches from the $j$ th cell to the $j+1$ st cell; specifically,

$$
d_{s, j}=n_{r, j} d_{r}+n_{n, j} d_{n}
$$

where $n_{r, j}\left(n_{n, j}\right)$ is the number of messages delivered through uplink DPCCH (ATM network) for frame synchronization and $d_{r}\left(d_{n}\right)$ is the expected transmission delays in the air interface (ATM network). For MOFC-III, it is clear that $d_{s, j}=4 d_{r}$ (see Fig. 3). Based on the modeling technique developed in [15], Fig. 4 plots the $d_{s, j} / d_{n}$ curves for the four frame-synchronization schemes. In this figure, $\lambda$ is the downlink packet frame arrival rate, $1 / \mu$ is the average interval that the UE connects to a serving cell, and $j$ represents the $j$ th serving cell switching performed by the UE for a downlink-transmission session. The figure indicates that MOFC outperforms BOFC, NOFC, and COFC. When $\lambda / \mu$ becomes large (i.e., more packet frames are received by the UE at a cell) or $j$ becomes large (i.e., more serving cell switching performed by the UE), the improvement becomes more significant. 


\section{CONCLUSION}

This paper described an overflow-control scheme with module count to support UMTS HSDPA mechanism specified in 3GPP TS 25.308 and TR 25.950. We first introduced HSDPA and then discussed the issues regarding buffer overflow and long frame-synchronization delay, which were not resolved in 3GPP TS 25.308 and TR 25.950. Specifically, when the UE switches the wireless link from the old serving cell to the new serving cell, the size of frame-synchronization information may exceed the capacity of an uplink DPCCH message. In this case, this information must be carried through multiple uplink DPCCH messages, which results in a long delay for frame synchronization. To resolve the above issues, we proposed the MOFC scheme. Our scheme guarantees that the information needed for frame synchronization is carried by one uplink DPCCH message delivery. Also, when the buffer of a nonserving cell is full, the previously received packet frames in the buffer can be safely dropped and after the UE has switched wireless link to the new serving cell, no packet frames are lost. We have proved that the MOFC scheme is correct. Then, we used numerical examples to illustrate the improvement of MOFC over several previously proposed schemes. Our study indicated that MOFC may significantly outperform the previously proposed schemes. The MOFC scheme is pending U.S. and R.O.C. patents.

\section{APPENDIX NOTATION}

The notation used in this paper is given below.

- $B_{1}\left(B_{2}\right)$ : buffer size of the serving cell (nonserving cell).

- $C R_{i}\left(C R_{s}\right)$ : counter maintained by the nonserving cell $\mathrm{Cell}_{i}$ (serving cell Cell ${ }_{s}$ ). This counter records the number of received packet frames that are sent from the RNC.

- $C S_{i}\left(C S_{s}\right)$ : counter maintained by the nonserving cell $\mathrm{Cell}_{i}$ (serving cell Cell $l_{s}$ ). This counter records the number of packet frames that have been processed by $\mathrm{Cell}_{i}\left(\mathrm{Cell}_{s}\right)$. For $\mathrm{Cell}_{i}, C S_{i}$ is the number of packet frames deleted from the buffer. For $\mathrm{Cell}_{s}, C S_{s}$ is the number of packet frames that have been received by the UE.

- $K_{i}\left(K_{s}\right)$ : number of packet frames currently stored in the buffer of the nonserving cell Cell ${ }_{i}$ (the serving cell Cell ${ }_{s}$ ).

- $C S_{\mathrm{RNC}}$ : counter maintained by the RNC to record the number of packet frames that have been received by the serving cell.

- $C R_{\mathrm{UE}}$ : counter maintained by the $\mathrm{UE}$ to count the number of the received packet frames (which are sent from $\mathrm{Cell}_{s}$ ).

- $N_{i}$ : maximum number of packet frames that can be accommodated in the buffer of $\mathrm{Cell}_{i}$.

- $N^{*}$ : maximum value of the number that can be carried through one uplink DPCCH message.

- $w$ : window size of the window-based flow-control algorithm exercised for downlink transmission from the RNC to the serving cell.

\section{ACKNOWLEDGMENT}

The authors would like to thank the anonymous reviewers: their comments have significantly improved the quality of this paper.

\section{REFERENCES}

[1] "3GPP. 3rd Generation Partnership Project," Tech. Spec. Group Radio Access Network. High-speed downlink packet access. Overall UTRAN description, Release 5. Tech. Rep. 3G TS 25.855 version 5.0.0 (2001-10), 2001.

[2] "3GPP. 3rd Generation Partnership Project," Tech. Spec. Group RadioAccess Network. Physical layer aspects of UTRA high-speed downlink packet access, Release 4. Tech. Rep. 3G TR 25.848 version 4.0.0 (2001-03), 2001.

[3] "3GPP. 3rd Generation Partnership Project," Tech. Spec. Group Radio Access Network. UTRA high-speed downlink packet access, Release 4. Tech. Rep. 3G TR 25.950 version 4.0.0 (2001-03), 2001.

[4] "3GPP. 3rd Generation Partnership Project," Tech. Spec. Group Radio Access Network. High-speed downlink packet access, overall description, Stage 2, Release 5. Tech. Rep. 3G TS 25.308 version 5.4.0 (2002-03), 2002.

[5] "3GPP. 3rd Generation Partnership Project," Tech. Spec. Group Radio Access Network. Radio-interface protocol architecture, Release 5. Tech. Rep. 3G TS 25.301 version 5.2.0 (2002-09), 2002.

[6] "3GPP. 3rd Generation Partnership Project," Tech. Spec. Group Radio Access Network. UTRAN iub interface, general aspects and principles, Release 5. Tech. Rep. 3G TS 25.430 version 5.2.0 (2002-09), 2002.

[7] “3GPP. 3rd Generation Partnership Project," Tech. Spec. Group Services and Systems Aspects, general packet radio service (GPRS), service description, Stage 2. Tech. Rep. 3G TS 23.060 version 6.1.0 (2003-06), 2003.

[8] A. Das, F. Khan, A. Sampath, and H.-J. Su, "Performance of hybrid ARQ for high speed downlink packet access in UMTS," in Proc. IEEE Vehicular Technology Conf. (VTC'01), vol. 4, 2001, pp. 2133-2137.

[9] P. Frenger, S. Parkvall, and E. Dahlman, "Performance comparison of HARQ with chase combining and incremental redundancy for HSDPA," in Proc. IEEE Vehicular Technology Conf. (VTC'01), vol. 3, 2001, pp. $1829-1833$

[10] H. Holma and A. Toskala, "WCDMA and WLAN for 3G and beyond," IEEE Wireless Commun., vol. 9, pp. 14-19, Apr. 2002.

[11] — WCDMA for UMTS, 2nd ed. New York: Wiley, 2002.

[12] W. S. Jeon, D. G. Jeong, and B. Kim, "Design of packet transmission scheduler for high speed downlink packet access systems," in Proc. IEEE Vehicular Technology Conf. (VTC'02), vol. 3, 2002, pp. $1125-1129$.

[13] H. Kaaranen, S. Naghian, L. Laitinen, A. Ahtiainen, and V. Niemi, UMTS Networks: Architecture, Mobility and Services. New York: Wiley, 2001.

[14] T. Kawamura, K. Higuchi, Y. Kishiyama, and M. Sawahashi, "Comparison between multipath interference canceller and chip equalizer in HSDPA in multipath channel," in Proc. IEEE Vehicular Technology Conf. (VTC'02), vol. 1, 2002, pp. 459-463.

[15] P. Lin, Y.-B. Lin, and I. Chlamtac, "Modeling frame synchronization for UMTS high-speed downlink packet access," IEEE Trans. Veh. Technol., vol. 52, pp. 132-141, Jan. 2003.

[16] — " "Overflow control for UMTS high-speed downlink packet access," IEEE Trans. Wireless Commun., vol. 3, Jan. 2004.

[17] Lucent, Murray Hill, NJ, "ARQ Technique for HSDPA,", Tech. Rep. R2A010 021

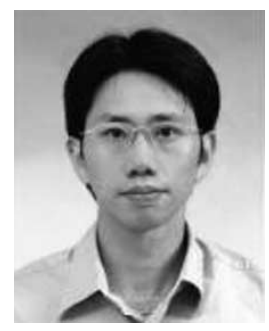

source Management.
Phone Lin (M'02) received the B.S.CSIE and $\mathrm{Ph} . D$. degrees from National Chiao Tung University, Taiwan, R.O.C., in 1996 and 2001, respectively.

In 2001, he was appointed an Assistant Professor in the Department of Computer Science and Information Engineering (CSIE), National Taiwan University, R.O.C. His current research interests include personal communications services, wireless Internet, and performance modeling.

Dr. Lin was a Guest Editor for a IEEE WIRELESS Communications special issue on Mobility and Re- 


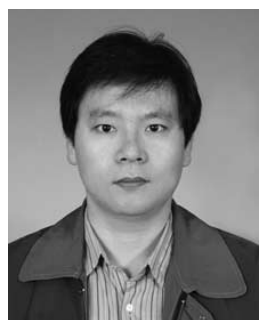

Yi-Bing Lin (M'95-SM'95-F'03) received the B.S.E.E. degree from National Cheng Kung University, Tainan, Taiwn, R.O.C., in 1983 and the Ph.D. degree in computer science from the University of Washington, Seattle, in 1990.

From 1990 to 1995, he was with the Applied Research Area, Bell Communications Research (Bellcore), Morristown, NJ. In 1995, he was appointed Professor, Department of Computer Science and Information Engineering (CSIE), National Chiao Tung University (NCTU), Hsinchu, Taiwan, R.O.C. In 1996, he was appointed Deputy Director of the Microelectronics and Information Systems Research Center at NCTU. From 1997 to 1999 , he was Chairman of CSIE at NCTU. He has published over 150 journal articles and more than 200 conference papers. He is the author of the book Wireless and Mobile Network Architecture (coauthored with Imrich Chlamtac; Wiley: New York, 2001). His current research interests include the design and analysis of personal communication services networks, mobile computing, distributed simulation, and performance modeling. He serves as a Consultant to many telecommunications companies, including FarEasTone and Chung Hwa Telecom.

Dr. Lin is a Senior Technical Editor of IEEE NETwORK. He is an Editor of IEEE TRANSACTIONS ON Wireless CoMmunications, IEEE Personal COMMUNICATIONS MAGAZINE, Computer Networks, International Journal of Communications Systems, ACM/Baltzer Wireless Networks, Computer Simulation Modeling and Analysis, and Journal of Information Science and Engineering. He is an Associate Editor of IEEE TRANSACTIONS ON VEHICULAR TECHNOLOGY and IEEE Communications Survey and Tutorials. He is an Area Editor of ACM Mobile Computing and Communication Review and a Columnist for ACM Simulation Digest. He has been a Guest Editor for the ACM/Baltzer MONET special issue on Personal Communications, the IEEE TRANSACTIONS ON COMPUTERS special issue on Mobile Computing, the IEEE TRANSACTIONS ON COMPUTERS special issue on Wireless Internet, and the IEEE COMMUNICATIONS MagaZine special issue on Active, Programmable, and Mobile Code Networking. He served as Program Chair for the Eighth Workshop on Distributed and Parallel Simulation and for the Second International Mobile Computing Conference. He also served as General Chair for the Ninth Workshop on Distributed and Parallel Simulation. He received the 1998, 2000, and 2002 Outstanding Research Awards from the National Science Council, R.O.C., and the 1998 Outstanding Youth Electrical Engineer Award from the Chinese Institute of Electrical Engineering (CIEE), R.O.C. He also received the NCTU Outstanding Teaching Award in 2002. He is an Adjunct Research Fellow of Academia Sinica and is Chair Professor at Providence University, Taichung, Taiwan, R.O.C

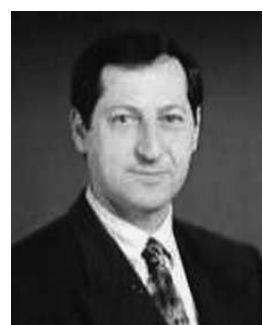

Imrich Chlamtac (M'86-SM'86-F'93) received the Ph.D. degree in computer science from the University of Minnesota, Minneapolis.

Since 1997, he held the Distinguished Endowed Chair in Telecommunications at the University of Texas at Dallas, Richardson, on a joint CS and EE appointment, from which he is currently on leave. He is currently the President of CreateNet, an international nonprofit research organization based in Europe, which consists of over two dozen universities, research centers, and member companies, including the University of Trento, Politecnico di Torino, Technical University of Berlin, Technion-The Israel Institute of Technology, the Technical University of Budapest, ICT-IRST, CNR, Microsoft, Hewlett-Packard, Nokia, EMC, Lucent, Marconi, and other industry leaders. He also holds the titles of the Sackler Professor at Tel-Aviv University, Israel, The Bruno Kessler Honorary Professor at the University of Trento, Italy, and University Professor at the Technical University of Budapest, Hungary. He has published over 300 refereed articles and is the coauthor of the first textbook on local area networks (Lexington Books, 1981, 1982, 1984) and of Mobile and Wireless Networks Protocols and Services (Wiley: New York, 2000), an Amazon.com bestseller and IEEE Network Magazine Editor's Choice. He serves as the founding Editor in Chief of the ACM/URSI/Kluwer Wireless Networks (WINET), the ACM/Kluwer Mobile Networks and Applications (MONET) journals, and the SPIE/Kluwer Optical Networks (ONM) magazine.

Dr. Chlamtac is a Fellow of the ACM, a Fulbright Scholar, and an IEEE Distinguished Lecturer. He is the winner of the 2001 ACM Sigmobile Annual Award and the IEEE ComSoc TCPC 2002 Award for contributions to wireless and mobile networks, and is the recipient of multiple Best Paper Awards in the areas of wireless and optical networks. He also serves on the Advisory Boards of multiple journals and publications. He is the Cofounder and Past President of CONSIP and $\mathrm{BCN}$ corporations. 